\title{
Linfoma relacionado a infección por virus de la inmunodeficiencia humana en un hospital público de Santiago, Chile
}

\author{
MARÍA ELENA CABRERA ${ }^{1}$, GUILLERMO SILVA ${ }^{\mathrm{a}}$, ANDRÉS SOTO ${ }^{\mathrm{b}}$, \\ RODRIGO ROSELLÓ ${ }^{\mathrm{b}}$, CONSTANZA CASTRO ${ }^{\mathrm{b}}$, VIRGINIA MARTÍNEZ ${ }^{2}$, \\ JUAN BALLESTEROS ${ }^{3}$, RODRIGO BLAMEY ${ }^{3}$, CLAUDIA GARREAUD ${ }^{3}$
}

\section{HIV-related lymphoma in a public hospital in Chile. Analysis of 55 cases}

Background: Cancer is the third cause of death in patients infected with human immunodeficiency virus (HIV) and lymphoma is the most common type. Aim: To describe the clinical characteristics, histology, risk factors and prognosis of these patients, in a Chilean public hospital in Chile. Material and Methods: Records of 55 patients ( 45 males) aged between 23 and 67 years with lymphoma and HIV positive serology, diagnosed between 1992-2008, were reviewed. Results: Six patients (11\%) had Hodgkin lymphoma (HL) and the rest, non-Hodgkin lymphoma (NHL). B-cell phenotype constituted $83.7 \%$ of NHL cases. The most common subtypes of all the lymphoma were diffuse large B cell lymphoma in 24 cases (43.6\%), Burkitt lymphoma in 12 cases (21.8\%), and plasmablastic lymphoma in 5 cases (9.1\%). Thirty five patients (64\%) underwent curative intended chemotherapy (CT) concomitantly with highly active antiretroviral therapy (HAART). Three year survival of the whole cohort was 27\%. By multivariate analysis, the most important prognostic factors for long term survival, were complete responses to $C T,(p<0.01)$ and a low international prognostic index (IPI) score for NHL, $(p=0.01)$. HAART, histologic subtype and $\mathrm{CD}_{4}$ lymphocyte count at diagnosis, did not influence survival. Conclusions: The most important prognostic factors for HIV patients with lymphoma, were achieving CR with CT and low IPI score. Prognosis remains poor, even with HAART therapy.

(Rev Med Chile 2012; 140: 243-250).

Key words: Antiretroviral therapy, highly active; HIV; Hodgkin disease; Lymphoma, non-Hodgkin.

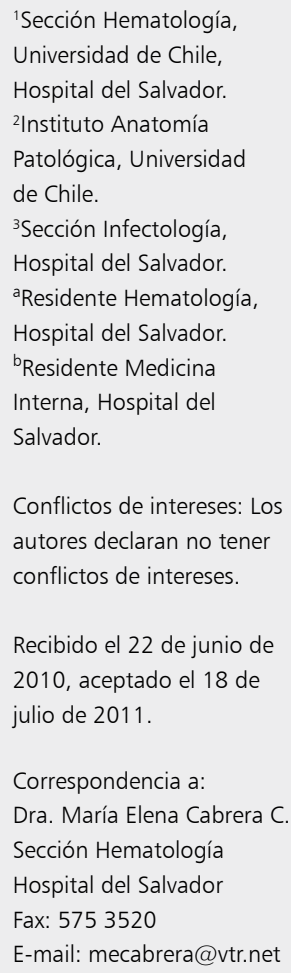

I as personas infectadas por el virus de la inmunodeficiencia humana (VIH) presentan una mayor morbimortalidad por cáncer, constituyendo en este grupo la tercera causa de muerte, a pesar de la efectividad de la terapia antirretroviral de alta eficacia (TARV). Varios estudios epidemiológicos han demostrado la disminución de infecciones oportunistas, sarcoma de Kaposi y linfoma primario cerebral y en menor proporción, linfoma no Hodgkin (LNH) y cáncer cervical. Sin embargo, se ha observado un incremento de neoplasias no asociadas a síndrome de inmunodeficiencia adquirida (SIDA) como el linfoma de Hodgkin (LH), cáncer anal, pulmón, cabeza y cuello y conjuntiva ${ }^{1-3}$. El uso de TARV ha mejorado el pronóstico de pacientes con linfoma debido a la reconstitución inmunológica ${ }^{4-8}$, aunque la sobrevida es significativamente menor que en pacientes seronegativos, debido a una menor tolerancia a drogas inmunosupresoras, mayor posibilidad de efectos adversos y mayor riesgo de infecciones ${ }^{1,9,10}$. 
El objetivo de este estudio fue analizar las características clínicas, tratamiento, sobrevida y factores pronósticos, de pacientes seropositivos con linfoma, diagnosticados y tratados en el Hospital del Salvador, centro de referencia del Area Oriente de la Región Metropolitana de Santiago, I, II y III regiones del país, donde se controlan alrededor de 1.500 pacientes con TARV. Además, se comparó con las características clínicas y sobrevida de pacientes con linfoma seronegativos, diagnosticados en el mismo período.

\section{Material y Método}

El estudio se basó en una evaluación de los pacientes con diagnóstico de linfoma y serología positiva para VIH, diagnosticados en los Servicios de Hematología e Infectología del Hospital del Salvador, entre 1992 y 2008 . El estudio de serología viral para VIH se realiza rutinariamente a todos los pacientes con diagnóstico de linfoma. El material histopatológico fue revisado por un solo patólogo (VM). Se utilizó la clasificación histológica $\mathrm{WHO}^{11} \mathrm{y}$ los pacientes fueron etapificados según el Protocolo de Linfoma del Programa de Cáncer del Adulto (PANDA) del Ministerio de Salud $(\text { Minsal })^{12}$, utilizando la clasificación clínica de Ann Arbor, que incluye historia clínica, examen físico, laboratorio de rutina, tomografías computadas de tórax, abdomen y pelvis, biopsia de médula ósea y consentimiento informado. Se realizó punción lumbar con citología de líquido cefalorraquídeo (LCR) a todos los pacientes con signos neurológicos o con linfoma de Burkitt (LB). El tratamiento del linfoma se basó en los esquemas recomendados por el protocolo del Minsal, manteniendo o iniciando el TARV, que se inició en Chile en 1997. La quimioterapia (QT) consistió en los esquemas de tratamiento del Minsal ${ }^{12}$ para LH, C-MOPP (ciclofosfamida, vincristina, procarbazina, prednisona) o ABVD (doxorrubicina, bleomicina, vinblastina, dacarbazina) según la fecha de diagnóstico y para LNH, CHOP (ciclofosfamida, doxorrubicina, vincristina, prednisona), COP (ciclofosfamida, vincristina, prednisona), esquema específico para LB o para linfoma primario de sistema nervioso central (SNC). Se utilizó ESHAP (etopósido, metilprednisolona, cisplatino, citarabina) como rescate. Algunos pacientes recibieron también radioterapia. La serología VIH (+) se confirmó por técnica de inmunofluorescencia indirecta (IFI) en el Instituto de Salud Pública. La etapa de infección por VIH se realizó de acuerdo con el criterio del CDC de 1993, en base al recuento de linfocitos CD4, la carga viral y la presencia de enfermedades oportunistas asociadas $^{13}$. La carga viral fue determinada por método NucliSens HIV-1 QT, y sus resultados fueron expresados en "copias de RNA viral/ml plasma", siendo 80 copias el límite inferior de detección. El recuento de $\mathrm{CD} 4$ se realizó por citometría de flujo.

La TARV antes del año 2000, consistió sólo en zidovudina con o sin didanosina. Desde el año 2000, consistió en zidovudina + lamivudina coformulados más efavirenz, modificada a abacavir + lamivudina coformulados más efavirenz, al conocerse el diagnóstico de linfoma. La remisión completa (RC) del linfoma se definió como ausencia de signos clínicos e imágenes por al menos 4 semanas; remisión parcial (RP) la reducción mayor a $50 \%$ y progresión $(\mathrm{P})$ la reducción menor a $50 \%$ o aumento de masas tumorales medibles.

Se evaluó las características relativas al linfoma y relativas a la infección por $\mathrm{VIH}$, que se muestran en la Tabla 1. El índice pronóstico internacional (IPI) ajustado a edad ${ }^{14}$ para LNH, incluye 3 factores de riesgo: etapa III y IV, performance status $2-4$ y LDH $>1,5$ veces el valor normal (bajo riesgo 0-1 factor, alto riesgo 2-3 factores).

Se estableció la asociación entre distintos subtipos de linfoma y características relacionadas al VIH, como desarrollo del linfoma durante la terapia o vírgenes a ésta y nivel de linfocitos CD4, utilizando el test $\chi^{2}$. La sobrevida global (SG) se calculó desde la fecha de inicio de tratamiento hasta la muerte por cualquier causa o fecha de último control. Ambas variables fueron analizadas por el test log rank y los resultados expresados con curvas de Kaplan-Meier. Los datos fueron analizados usando el programa estadístico Statistical Software Package for Social Sciences (SPSS versión 10.1). Los valores $p=0,05$ o menos se consideraron estadísticamente significativos. El modelo de riesgo proporcional de Cox se utilizó para determinar SG en análisis de multivarianza.

\section{Resultados}

\section{Características clínicas}

Entre 1992 y 2008, en el Hospital del Salvador, se diagnosticó linfoma a 840 pacientes, 132 con $\mathrm{LH}$ y 708 con LNH. De ellos, 6/132 (4,5\%) con LH y $49 / 708(6,9 \%)$ con LNH resultaron con serología 
Tabla 1. Características clínicas y de laboratorio de 55 pacientes con linfoma VIH positivo

\begin{tabular}{|c|c|c|}
\hline Variable & $\mathbf{n}$ & $\%$ \\
\hline Sexo, Varones & 45 & 82 \\
\hline $\begin{array}{l}\text { Contacto sexual }(n=40) \\
\text { Homo/bisexual } \\
\text { Heterosexual }\end{array}$ & $\begin{array}{r}31 \\
9\end{array}$ & $\begin{array}{l}78 \\
22\end{array}$ \\
\hline Edad, mediana años (rango) & 38 & $(23-67)$ \\
\hline $\begin{array}{l}\text { Etapa clínica }(\mathrm{n}=55) \\
\text { I-II } \\
\text { III-IV }\end{array}$ & $\begin{array}{l}20 \\
35\end{array}$ & $\begin{array}{l}36 \\
64\end{array}$ \\
\hline $\begin{array}{l}\text { Tiempo uso TARV previo desarrollo } \\
\text { linfoma }(n=22) \\
\quad \text { Media, meses (rango) }\end{array}$ & 15 & $(1-52)$ \\
\hline $\begin{array}{l}\text { Tiempo desde el diagnóstico de VIH } \\
\text { y linfoma } \\
\text { Media, años (rango) }\end{array}$ & 2,5 & $(0,0-19,0)$ \\
\hline $\begin{array}{l}\text { Deshidrogenasa láctica }>1,5 \text { veces } \\
\text { normal }(n=49)\end{array}$ & 37 & 75 \\
\hline $\mathrm{IPI}$ ae $=0>2(\mathrm{n}=49)$ & 32 & 65 \\
\hline Albúmina $<3,5 \mathrm{~g} / \mathrm{L}(\mathrm{n}=43)$ & 36 & 84 \\
\hline $\begin{aligned} \mathrm{CD} 4 & \times \mathrm{mm}^{3}(\mathrm{n}=49): \text { media (rango) } \\
& <100 \times \mathrm{mm}^{3}\end{aligned}$ & $\begin{array}{r}108 \\
23\end{array}$ & $\begin{array}{c}(2-575) \\
47\end{array}$ \\
\hline $\begin{array}{l}\text { Carga viral: copias RNA/ml }(n=40) \text { : } \\
\text { media (rango) }\end{array}$ & 120.000 & $(0-2.400 .000)$ \\
\hline
\end{tabular}

TARV: terapia anti-retroviral; IPI ae: indice pronóstico internacional ajustado a edad.

Tabla 2. Distribución de los subtipos histológicos en $\mathbf{5 5}$ casos de linfoma VIH positivo, de acuerdo a su frecuencia

\begin{tabular}{|lcc|}
\hline Subtipo & $\begin{array}{c}\text { n casos } \\
(\mathbf{n}=\mathbf{5 5})\end{array}$ & $\begin{array}{c}\text { \% del total } \\
\text { de casos }\end{array}$ \\
Linfoma de Hodgkin & 6 & 10,9 \\
Esclerosis nodular & 2 & 3,6 \\
Celularidad mixta & 2 & 3,6 \\
Depleción linfocitaria & 1 & 1,8 \\
Inclasificable & 1 & 1,8 \\
Linfoma no Hodgkin & 49 & 89,1 \\
Linfoma no Hodgkin de estirpe B & 41 & 74,5 \\
Difuso células grandes B & 24 & 43,6 \\
Linfoma de Burkitt & 12 & 21,8 \\
Linfoma plasmablástico & 5 & 9,1 \\
Linfoma no Hodgkin de estirpe T/NK & 8 & 14,5 \\
* ACG, alk (-) & 4 & 7,3 \\
T/NK nasal & 2 & 3,6 \\
T periférico & 1 & 1,8 \\
Linfoblástico T & 1 & 1,8 \\
\hline
\end{tabular}

*ACG,alk (-): anaplástico de células grandes alk (-).
VIH positiva. El diagnóstico de VIH en 31 casos $(56,4 \%)$, se realizó entre 0 y 19 años antes de la aparición del linfoma (media 2,5 años). En cambio en 24 casos $(43,6 \%)$ ambos diagnósticos fueron simultáneos. Las características clínicas y de laboratorio se presentan en la Tabla 1. El linfoma difuso de células grandes $\mathrm{B}$ (LDCGB) representó el subtipo más común $43,6 \%$, seguido del LB $21,8 \%$, linfoma T y T/NK 14,5\%, LH 10,9\% y linfoma plasmablástico (LP) 9,1\% (Tabla 2). El $83,6 \%$ de los LNH (41/49), correspondió a estirpe B. Dieciséis casos (29\%) tenían enfermedad extranodal localizada al diagnóstico. Los sitios extranodales comprometidos con mayor frecuencia fueron: gastrointestinal 7, médula ósea 6, maxilar/ cavidad oral/amígdala 6 , óseo 5 , riñón 4 , sistema nervioso central 4 y anorrectal tres. La mayoría de los pacientes con $\mathrm{LNH}$ presentaron IPI de alto riesgo, albúmina baja, y aproximadamente la mitad CD4 < $100 \mathrm{~mm}^{3}$ (Tabla 1). Sólo en 1 caso de linfoma T, se estudió la serología para HTLV-1 que resultó negativa. En las Tablas 3 y 4 se muestra la comparación de pacientes con linfoma VIH positivo y negativo.

\section{Terapia antiretroviral}

Cuarenta pacientes $(73 \%)$ recibieron TARV. Dos de ellos recibieron sólo zidovudina o asociado a didanosina, ya que el diagnóstico de VIH positivo fue previo a 1998. Veintidós de ellos recibían esta terapia al momento del diagnóstico del linfoma, con una mediana de 12 meses (rango 1-52 meses), mientras que los otros 18 (45\%), la iniciaron junto con la QT del linfoma. Quince casos (27\%) no recibieron terapia: 11 por fallecimiento precoz, tres por rechazo $\mathrm{y}$ uno por diagnóstico postmortem.

\section{Tratamiento antineoplásico y respuesta}

Linfoma de Hodgkin. Cinco de 6 pacientes $(83 \%)$ recibieron QT con intención curativa, $\operatorname{ABVD}(\mathrm{n}=4)$ y C-MOPP $(\mathrm{n}=1)$. El otro no fue tratado. Cuatro de $5(80 \%)$ lograron RC. Sólo uno sobrevive, dos recayeron (a los 14 y 36 meses de diagnóstico) y el otro falleció 14 años después por cáncer pulmonar, en remisión del linfoma. 
Tabla 3. Comparación de las características clínicas de 132 pacientes con linfoma de Hodgkin, VIH positivo y VIH negativo

\begin{tabular}{|c|c|c|c|}
\hline Variables & $\begin{array}{c}\text { VIH } \\
\text { positivo } \\
(n=6)\end{array}$ & $\begin{array}{c}\text { VIH } \\
\text { negativo } \\
(n=126)\end{array}$ & $\mathbf{p}$ \\
\hline $\begin{array}{l}\text { Edad, mediana años } \\
\text { (rango) }\end{array}$ & $34(30-41)$ & $28(16-82)$ & 0,31 \\
\hline Sexo masculino, n (\%) & $6 / 6(100)$ & $54 / 126(43)$ & 0,007 \\
\hline $\begin{array}{c}\text { Etapa clínica, n (\%) } \\
\text { I y II } \\
\text { III y IV }\end{array}$ & $\begin{array}{l}1(17) \\
5(83)\end{array}$ & $\begin{array}{l}60(48) \\
66(52)\end{array}$ & 0,14 \\
\hline $\begin{array}{l}\text { Histología, n (\%) } \\
\text { EN } \\
\text { CM } \\
\text { DL } \\
\text { PL } \\
\text { Inclasificable }\end{array}$ & $\begin{array}{l}2(33) \\
2(33) \\
1(17) \\
0 \quad(0) \\
1(17)\end{array}$ & $\begin{array}{rr}76 & (58) \\
41 & (32) \\
7 & (5) \\
6 & (5) \\
0 & (0)\end{array}$ & $0,48^{*}$ \\
\hline SG 3 años, \% & 50 & 80 & 0,21 \\
\hline
\end{tabular}

$\mathrm{EN}=$ esclerosis nodular; $\mathrm{CM}=$ celularidad mixta; $\mathrm{DL}=$ depleción linfocitaria; $\mathrm{PL}=$ predominio linfocítico; $\mathrm{LH}=$ linfoma de Hodgkin; $\mathrm{SG}=$ sobrevida global. * se agruparon las histologías debido al bajo número de casos VIH positivo.

Tabla 4. Comparación de las características clínicas de 708 pacientes con linfoma no Hodgkin, VIH positivo y VIH negativo

\begin{tabular}{|c|c|c|c|}
\hline Variable & $\begin{array}{c}\text { VIH } \\
\text { positivo } \\
(n=49)\end{array}$ & $\begin{array}{c}\text { VIH } \\
\text { negativo } \\
(n=659)\end{array}$ & $\mathbf{p}$ \\
\hline $\begin{array}{l}\text { Edad, mediana años } \\
\text { (rango) }\end{array}$ & $40(23-67)$ & $62(15-94)$ & 0,31 \\
\hline Sexo masculino, n (\%) & $39 / 49(80)$ & $316 / 659(48)$ & $<0,0001$ \\
\hline $\begin{array}{c}\text { Etapa clínica, n (\%) } \\
\text { I y II } \\
\text { III y IV }\end{array}$ & $\begin{array}{l}19(39) \\
30(61)\end{array}$ & $\begin{array}{l}283(43) \\
376(57)\end{array}$ & 0,33 \\
\hline $\begin{array}{l}\text { Histología, n (\%) } \\
\text { LDCGB } \\
\text { Burkitt } \\
\text { T/NK } \\
\text { Plasmablástico } \\
\text { Otros }\end{array}$ & $\begin{array}{l}24(49) \\
12(25) \\
8(16) \\
5(10) \\
0\end{array}$ & $\begin{array}{r}312(47) \\
18(3) \\
126(19) \\
0,0(0) \\
203(31)\end{array}$ & $\begin{aligned} & 0,47 \\
< & 0,0001 \\
& 0,39 \\
< & 0,0001\end{aligned}$ \\
\hline $\begin{array}{c}\text { SG } 3 \text { años, \% } \\
\text { LDCGB } \\
\text { Burkitt } \\
\text { T/NK } \\
\text { LP }\end{array}$ & $\begin{array}{l}28,6 \\
20 \\
16,6 \\
50\end{array}$ & $\begin{array}{c}54 \\
49 \\
32,6 \\
0\end{array}$ & $\begin{array}{l}0,0004 \\
0,0006 \\
0,01\end{array}$ \\
\hline
\end{tabular}

$\mathrm{LDCGB}=$ linfoma difuso células grandes $\mathrm{B} ; \mathrm{LP}=$ linfoma plasmablástico; SG = sobrevida global.
Linfoma no Hodgkin. Treinta de 49 pacientes $(61 \%)$ recibieron QT con intención curativa. De los 24 casos con LDCGB, 14 recibieron QT, $\mathrm{CHOP}(\mathrm{n}=11)$, $\mathrm{COP}(\mathrm{n}=2)$ y esquema específico para linfoma primario de SNC $(n=1)$. Sólo 6 lograron RC $(25 \%)$ y cuatro se mantienen en remisión (39-72 meses). De los 12 casos con $\mathrm{LB}, 5$ recibieron QT esquema específico para LB $(n=2)$, COP $(\mathrm{n}=2)$ y CHOP $(\mathrm{n}=1)$. Sólo dos lograron RC (40\%), ambos con esquema de LB y uno se mantiene en remisión (51 meses). De los cinco pacientes con LP, todos recibieron esquema CHOP, tres lograron RC (60\%) y dos se mantienen en remisión (6-12 meses). De los 8 pacientes con linfoma $\mathrm{T}$ y T/NK, 6 recibieron $\mathrm{CHOP}$, sólo uno logró $\mathrm{RC}(16,6 \%)$ y se mantiene en remisión (44 meses). Dos pacientes fueron tratados con esquema de rescate ESHAP, por recaída o enfermedad progresiva, sin respuesta. Siete de 35 pacientes $(20 \%)$ recibieron además radioterapia, tres con intención curativa por enfermedad residual localizada (maxilar, SNC y cervical) y los otros cuatro con intención paliativa.

Diecinueve de 49 casos con LNH, no recibieron QT, por mal estado general $(\mathrm{n}=13)$, rechazo $(\mathrm{n}=3)$ y diagnóstico post mortem $(\mathrm{n}=1)$. Dos, fueron tratados sólo con TARV, logrando remisión del linfoma en uno de ellos (72 meses).

\section{Sobrevida y pronóstico}

La SG a 3 años de pacientes con LH fue 50\%, LDCGB 28,6\%, LB 20\%, linfomas T $16,6 \%$ y LP $50 \%$, sin diferencia significativa entre los subtipos histológicos $(\mathrm{p}=0,54)$, Figura 1. El seguimiento medio fue 62 meses (1 mes-14 años). La sobrevida media de los pacientes no tratados fue 1,5 meses.

En cambio, la SG a 3 años de pacientes VIH negativos con LH fue 80,6\%, LDCGB $54 \%$, LB $49 \%$ y linfomas T 32,6\% (Tablas 3 y 4). No se diagnosticó LP en pacientes seronegativos.

El análisis univariado de diferentes factores pronósticos demostró que los factores más importantes de sobrevida fueron lograr $\mathrm{RC}$ del linfoma $(\mathrm{p}<0,0001)$ y el IPI bajo en LNH $(p=0,0009)$. De menor importancia, pero aún significativo, fueron la edad menor 


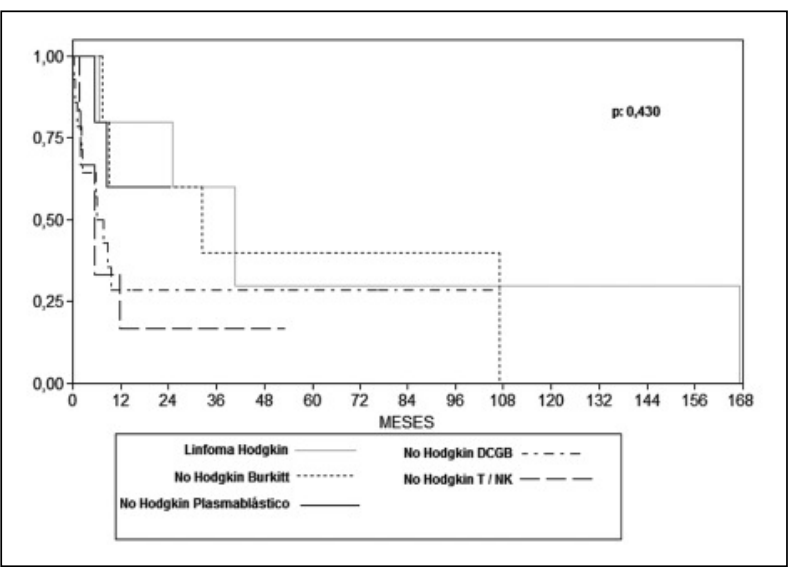

Figura 1. Sobrevida global según subtipo histológico de linfoma en 35 pacientes VIH positivo tratados con quimioterapia con intención curativa.

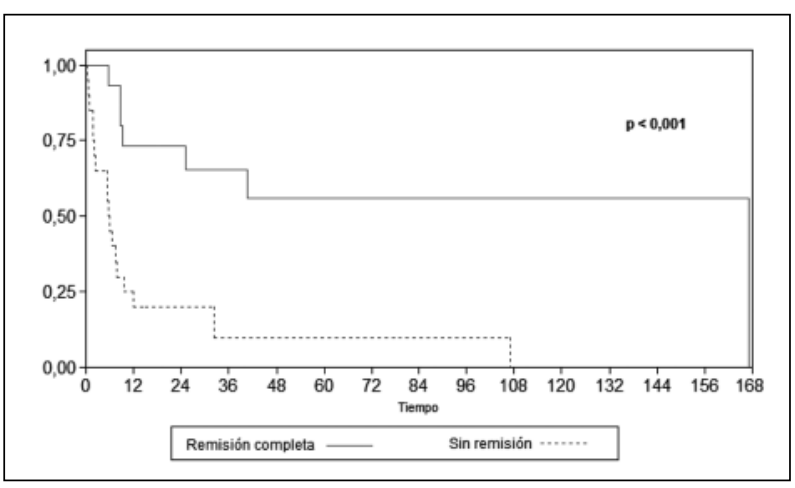

Figura 2. Sobrevida global según respuesta al tratamiento del linfoma en 35 pacientes $\mathrm{VIH}$ positivo tratados con quimioterapia con intención curativa: remisión completa o no.

de 40 años $(\mathrm{p}=0,02)$ y la etapa clínica localizada $(\mathrm{p}=$ $0,03)$. No hubo diferencia significativa entre los subtipos histológicos $(\mathrm{p}=0,54)$, nivel de linfocitos $\mathrm{CD} 4<$ ó $>100$ células $/ \mathrm{mm}^{3}(\mathrm{p}=0,7)$ o si el linfoma fue diagnosticado en el transcurso de TARV o vírgenes a ella. Cuarenta y uno de 55 pacientes han fallecido $(74,5 \%)$. La causa de muerte estuvo relacionada con linfoma progresivo en 22 casos, complicaciones relacionadas a infección VIH en 13, ambas causas en cinco y cáncer pulmonar un caso.

\section{Comparación de pacientes con linfoma VIH positivo y negativo}

La comparación de pacientes con $\mathrm{LH}$, VIH positivo y negativo, demostró que no hay diferencia estadísticamente significativa para edad, etapa clínica, histología ni SG, sólo hay diferencia respecto a predominio de sexo masculino en el grupo VIH positivo (Tabla 3).

La comparación de pacientes con $\mathrm{LNH}$, VIH positivo y negativo, demostró que no hay diferencia estadísticamente significativa para edad, etapa clínica, subtipo histológico LDCGB y TN/K. En cambio, hubo diferencia respecto a mayor frecuencia de LB y LP en el grupo VIH positivo y menor SG en todos los subtipos histológicos del grupo VIH positivo (Tabla 4).

\section{Discusión}

En este estudio demostramos que la asociación de linfoma y VIH continúa siendo factor de mal pronóstico, en comparación con pacientes con linfoma seronegativos.

La mayor diferencia epidemiológica con éstos, se observó en la distribución de los subtipos histológicos, especialmente en $\mathrm{LNH}$, y fue similar a la descrita en la literatura. El LDCGB fue el doble más común que el LB y este último, constituyó la cuarta parte de todos los subtipos histológicos. En cambio, en casos VIH negativos, el LB alcanza apenas 3\%. La otra gran diferencia, fue la presencia del LP, una variedad de linfoma de células $B$, descrito específicamente en pacientes VIH positivo, por primera vez en $1997^{15}$. En nuestra casuística alcanzó $10 \%$, en cambio para Carbone $\mathrm{A}^{16}$ éste constituyó sólo $2,6 \%$ de todos los $\mathrm{LNH}$ asociados a VIH. Al igual que en la literatura, la mitad de nuestros casos, se presentó en la cavidad oral ${ }^{15-17}$.

Destaca la alta incidencia de linfomas $\mathrm{T}$ en nuestra serie, $16 \%$ de los LNH, concordante con el $17,4 \%$ de otro estudio chileno de los mismos autores (MEC), con neoplasias linfoproliferativas diseminadas en pacientes VIH negativo ${ }^{23}$. Esta cifra elevada, se observó también en LNH seronegativos diagnosticados en este período (Tabla 4), la mayoría de los cuales son HTLV-1 negativos. Por tanto, esta infección no está asociada a la mayoría de los linfomas T. El único trabajo publicado en Chile de 14 pacientes, no describe linfomas $\mathrm{T}^{18}$. Existen pocas publicaciones de linfomas T en pacientes VIH positivos. En la mayoría, la cifra es inferior, $3 \%$ en una serie de Los Angeles, California ${ }^{19}$, 3,8\% de Madrid ${ }^{20}$ y $1 \%$ de 
México $^{21}$. La única publicación con una frecuencia más elevada se observó en Perú, en el cual 9/33 casos (27\%) eran de estirpe $\mathrm{T}^{22}$. El fenotipo $\mathrm{T}$ es un factor pronóstico adverso en comparación con el fenotipo $\mathrm{B}$, según el grupo español de $\operatorname{SIDA}^{20}$, al igual que en inmunocompetentes.

El diagnóstico de VIH y linfoma se realizó en forma concomitante en aproximadamente la mitad de nuestros casos, lo que revela que aún hay una demora en la pesquisa de seropositividad. Es decir, la serología positiva se hace evidente recién, con la presencia del cáncer. Por el contrario, un estudio del Reino Unido ${ }^{24}$, reveló que sólo en un pequeño porcentaje (11\%), el linfoma se diagnosticó en forma simultánea con el VIH. El descenso de la incidencia de LNH descrito en los últimos años en individuos infectados con VIH en países desarrollados, al parecer está en relación a un inicio más precoz de la terapia antirretroviral y a un acceso más amplio a exámenes de serología viral, situación menos favorable en nuestro país. El presente estudio demuestra la necesidad de educar a la población en riesgo y mejorar el acceso a estos exámenes, para un diagnóstico más precoz de la infección viral.

Sólo 2 factores se asociaron significativamente a mejor sobrevida, la RC con QT y el IPI bajo. Estudios al respecto han demostrado también la importancia de lograr $\mathrm{RC}$ en $\mathrm{LH}^{7,25}$ y LNH ${ }^{6,8}$. Así, Wolf T. et al, en Frankfurt ${ }^{26}$, demostraron que el único factor pronóstico independiente asociado a mayor sobrevida, fue lograr la RC, en 214 casos de LNH, por sobre una adecuada respuesta virológica. El otro factor pronóstico independiente fue el IPI en LNH, ya que un score bajo (0-1) está asociado a mejor sobrevida y varios estudios han destacado la importancia de su aplicación ${ }^{8,17,27,28}$.

Se ha descrito que el nivel de linfocitos CD4 es uno de los factores pronósticos importantes, al diagnóstico del linfoma. Algunos estudios han demostrado que un nivel $<100 \mathrm{x} \mathrm{mm}^{3}$ es un factor adverso independiente ${ }^{27,28}$. Esta asociación adversa no fue observada en nuestro estudio.

El uso rutinario de TARV ha mejorado significativamente el pronóstico de los pacientes VIH positivos con $\mathrm{LH}^{7,25,29}$, en comparación con el período previo a su uso, utilizando QT estándar como ABVD. La razón es la mejoría de la función inmune y mayor tolerancia a las drogas inmunosupresoras. $\mathrm{Al}$ respecto, Hentrich M. et $\mathrm{al}^{7}$ demostraron una mejoría significativa de SG a 2 años en pacientes que recibieron TARV, comparado con aquellos que no la recibieron, $74 \%$ y $30 \%$, respectivamente $(\mathrm{p}<0,001)$. Mejor resultado obtuvo el grupo español, con SG a 5 años de $76 \%{ }^{29}$. En cambio en nuestro estudio, aún con TARV, la SG a 3 años fue $50 \%$, inferior a pacientes VIH negativos de nuestro país y tratados también, en hospitales públicos (30), aunque no significativo (Tabla 3 ).

En LNH, el uso de TARV ha mejorado el pronóstico principalmente en LDCGB $^{6,26}$, utilizando QT estándar como CHOP. Por ejemplo, Lim ST. et $\mathrm{al}^{6}$ comunicaron una mejoría notable en la sobrevida media, comparando la era pre y post terapia de alta eficacia, de 8,3 meses a 43,2 meses, respectivamente $(\mathrm{p}<0,0003)$. En cambio, el mismo estudio no demostró este beneficio en LB, con sobrevida media sólo de 6,4 y 5,4 meses, pre y post TARV, respectivamente. En nuestro estudio, ninguno de los dos subtipos de LNH pareció beneficiarse con TARV, observándose SG significativamente inferior en ambos grupos, en comparación con pacientes seronegativos (Tabla 4).

No hay consenso aún sobre el tratamiento de QT más apropiado del linfoma en el paciente VIH positivo, aunque la mejoría en la sobrevida del LDCGB, se ha observado utilizando los mismos esquemas de QT estándar tipo CHOP, sólo con la introducción de TARV. Se ha utilizado esquemas intensos en pacientes con LB, como HyperCVAD ${ }^{31}$ o CODOX-M/IVAC ${ }^{32}$, demostrando tasas de sobrevida similar a la de pacientes seronegativos (SLE a 2 años 60\%) aunque en pequeño número de casos. Es probable que el pronóstico adverso de los pacientes con LB del estudio de Lim ST et $\mathrm{al}^{6}$ se explique porque en este estudio se utilizó terapia CHOP. La asociación de rituximab al CHOP, no ha logrado mejorar la sobrevida en LDCGB asociado a $\mathrm{VIH}^{33}$, debido a mayor mortalidad por infecciones, especialmente en pacientes con recuento de $\mathrm{CD} 4<50 \mathrm{xmm}^{3}$. En cambio en $\mathrm{LB}$, la asociación de rituximab con altas dosis de QT, demostró una elevada sobrevida, similar a pacientes seronegativos, $73 \%$ y $82 \%$, respectivamente ${ }^{34}$. Se ha utilizado incluso, trasplante autólogo de progenitores hematopoyéticos ${ }^{35}$, en pacientes con recaídas quimiosensibles. Faltan más estudios para plantear el uso rutinario de rituximab en LB.

La respuesta inmunológica y virológica a la terapia de alta eficacia, no fue evaluada en nuestro estudio, como tampoco se consignó la toxicidad a las drogas antineoplásicas. Parece apropiado hacerlo en el futuro, para tener una evaluación 
más precisa de la evolución y tolerancia a drogas inmunosupresoras, ya que la eficacia a la terapia está asociada a mejor sobrevida ${ }^{36}$.

En conclusión, los factores más importantes de sobrevida de los pacientes con linfoma asociado a VIH, fueron la RC del linfoma y el IPI bajo en LNH. En cambio, no hubo diferencia en SG entre los distintos subtipos histológicos de linfoma. La comparación de SG con pacientes seronegativos de nuestro país, demostró ser significativamente inferior en LNH, no así en LH. Dado que la cohorte de pacientes descritos pertenece a un centro hospitalario y de derivación del sistema público, la muestra podría ser representativa de lo que ocurre en Chile. Es necesario, sin embargo, mejorar la oportunidad diagnóstica en VIH y optimizar el acceso a la salud, para poder lograr un impacto real en el pronóstico y la sobrevida. Además es fundamental la realización de mayores investigaciones en un futuro, idealmente en forma multicéntrica, para tener un mayor conocimiento respecto al comportamiento clínico y evolución de estos pacientes.

Agradecimientos. Agradecemos al Sr. Gabriel Cavada, Gonzalo Riadi y Dr. Mauricio Salinas, por su apoyo en el análisis estadístico y al Dr. Fernando Florenzano por un análisis crítico del trabajo.

\section{Referencias}

1. HIV infection and cancer in the era of highly active antiretroviral therapy (Review). Oncol Rep 2007; 17: 1121-6.

2. Bennet F, Chene G. Evolving epidemiology of malignancies in HIV. Curr Opin Oncol 2008; 20: 534-40.

3. Powles T, Robinson D, Stebbing J, Shamash J, Nelson M, Gazzard B, et al. Highly active antiretroviral therapy and the incidence of non-AIDS-defining cancers in people with HIV infection. J Clin Oncol 2009; 27: 88490.

4. Navarro JT, Ribera JM, Oriol A, Romeu J, Sirera G, Mate JL, et al. Favorable impact of virological response to highly active antiretroviral therapy on survival in patients with AIDS-related lymphoma. Leuk Lymphoma 2002; 43: 1837-42.

5. Hoffmann C, Wolf E, Fatkenheuer G, Buhk T, Stoehr A, Plettenberg A, et al. Response to highly active antiretroviral therapy strongly predicts outcome in patients with AIDS-related lymphoma. AIDS 2003; 17: 1521-9.
6. Lim ST, Karim R, Nathwani BN, Tulpule A, Espina B, Levine AM. AIDS-related Burkitt's lymphoma versus diffuse large-cell lymphoma in the pre-highly active antiretroviral therapy (HAART) and HAART eras: significant differences in survival with standard chemotherapy. J Clin Oncol 2005; 23: 4430-8.

7. Hentrich M, Maretta L, Chow KU, Bogner JR, Schürmann D, Neuhoff P, et al. Highly active antiretroviral therapy (HAART) improves survival in HIV-associated Hodgkin's disease: results of a multicenter study. Ann Oncol 2006; 17: 914-9.

8. Mournier N, Spina M, Gabarre J, Raphael M, Rizzardini G, Golfier JB, et al. AIDS-related non-Hodgkin lymphoma: final analysis of 485 patients treated with risk-adapted intensive chemotherapy. Blood 2006; 107: 3832-40.

9. Spano JP, Costagliola D, Katlama C, Mournier N, Oksenhendler E, Khayat D. AIDS-related malignancies: state of the art and therapeutic challenges. J Clin Oncol 2008; 26: 4834-42.

10. Chao C, Xu L, Abrams D, Leyden W, Horberg M, Towner W, et al. Survival of non-Hodgkin lymphoma patients with and without HIV infection in the era of combined antiretroviral therapy. AIDS 2010; 24:176570.

11. Swerdlow SH, Campo E, Harris NL, Jaffe ES, Pileri SA, Stein $\mathrm{H}$, et al. Who classification of tumours of Haematopoietic and lymphoid tissues. IARC Press: Lyon. 2008.

12. Protocolos Programa Nacional Cancer del Adulto. PANDA. Ministerio de Salud. 2008.

13. 1993 Revised classification system for HIV infection and expanded surveillance case definition for AIDS among adolescents and adults. MMWR Recomm Rep 1992; 41: 1-19.

14. Shipp MA, Harrington D, Anderson J, et al. Development of a predictive model for I lymphoma: The international NHL prognostic factors project. N Engl J Med 1993; 329: 987-94.

15. Delecluse HJ, Anagnostopoulos I, Dallenbach F, Hummel M, Marafioti T, Schneider U, et al. Plasmablastic lymphomas of the oral cavity: a new entity associated with the human immunodeficiency virus infection. Blood 1997: 89: 1413-20.

16. Carbone A. AIDS-related non-Hodgkin's lymphomas: From pathology and molecular pathogenesis to treatment. Human Pathology 2002; 33: 392-404.

17. Raviele R, Pruneri G, Maiorano E. Plasmablastic lymphoma: a review. Oral diseases 2009; 15: 38-45.

18. Osorio G, Montenegro C. Linfomas asociados a infección por virus de inmunodeficiencia humana en un complejo hospitalario de la Región Metropolitana, 
Chile: 1990-2002. Reporte de 14 casos y revisión. Rev Chil Infect 2007; 24: 117-24.

19. Arzoo KK, Bu X, Espina BM, Seneviratne L, Nathwani B, Levine AM. T-cell lymphomas in HIV infected patients. J Acquir Immune Defic Syndr 2004; 36: 1020-7.

20. Miralles P, Berenguer J, Ribera JM, Rubio R, Mahillo B, Téllez MJ, et al. Grupo de Estudio del SIDA Register of Systemic AIDS-Related Lymphomas. Prognosis of AIDS-related systemic non-Hodgkin lymphoma treated with chemotherapy and highly active antiretroviral therapy depends exclusively on tumor-related factors. J Acquir Immune Defic Syndr 2007; 44: 167-73.

21. Cornejo P, Volkow P, Aviles A, Calderon E. AIDS and non-Hodgkin's lymphoma. Experience at an oncological center in Mexico. Rev Invest Clin 2008; 60: 375-81.

22. Collins JA, Hernández AV, Hidalgo JA, Villena J, Sumire J, Delgado V, et al. Almenara Hospital AIDS Working Group. High proportion of T-cell systemic non-Hodgkin lymphoma in HIV-infected patients in Lima, Peru. J Acquir Immune Defic Syndr 2005; 40: 558-64.

23. Cabrera ME, Marinov N, Guerra C. Síndromes linfoproliferativos crónicos en Chile. Estudio prospectivo de 132 casos. Rev Med Chile 2003; 131: 291-98.

24. Bower M, Fisher M, Hill T, Reeves I, Walsh J, Orkin C, et al. for the UK CHIC Steering Committee. CD4 counts and the risk of systemic non-Hodgkin's lymphoma in individuals with HIV in the UK. Haematologica 2009; 94: 875-80.

25. Berenguer J, Miralles P, Ribera JM, Rubio R, Valencia E, Mahillo B, et al. Characteristics and outcome of AIDS-related Hodgkin lymphoma before and after the introduction of highly active antiretroviral therapy. J Acquir Immune Defic Syndr 2008; 47: 422-8.

26. Wolf T, Brodt HR, Fichtlscherer S, Mantzsch K, Hoelzer $\mathrm{D}$, Helm EB, et al. Changing incidence and prognostic factors of survival in AIDS-related non-Hodgkin's lymphoma in the era of highly active antiretroviral therapy (HAART). Leuk Lymphoma 2005; 46: 207-15.

27. Bower M, Gazzard B, Mandalia S, Newsom-Davis T, Thirlwell C, Dhillon T, et al. A prognostic index for systemic AIDS-related non-Hodgkin lymphoma treated in the era of highly active antiretroviral therapy. Ann Intern Med 2005; 16; 143: 265-73.

28. Simcock M, Blasko M, Karrer U, Bertisch B, Pless M, Blumer L, et al. Swiss HIV Cohort Study. Treatment and prognosis of AIDS-related lymphoma in the era of highly active antiretroviral therapy: findings from the Swiss HIV Cohort Study. Antivir Ther 2007; 12: 931-9.

29. Xicoy B, Ribera JM, Miralles P, Berenguer J, Rubio R, Mahillo B,et al. GESIDA Group; GELCAB Group. Results of treatment with doxorubicin, bleomycin, vinblastine and dacarbazine and highly active antiretroviral therapy in advance stage human immunodeficiency virus-related Hodgkin's lymphoma. Haematologic. 2007; 92: 191-8.

30. Cabrera ME, García H, Lois V, León A, Peña K, Rossle A, et al. Linfoma de Hodgkin en Chile. Experiencia de 15 años del Programa de Cáncer del Adulto. Rev Med Chile 2007; 135: 341-50.

31. Cortes J, Thomas D, Ríos A, Koller C, O’Brien S, Jeha S, et al. Hyperfractionated cyclophosfamide, vincristine, doxorubicin and dexamethasone and highly antirretroviral therapy for patients with acquired immunodeficiency sindrome-related Burkitt lymphoma/leukemia. Cancer 2002; 94: 1492-9.

32. Wang ES, Straus DJ, Teruya-Feldstein J, Qin J, Portlock C, Moskowitz C, et al. Intensive chemotherapy with cyclophosfamide, doxorubicin, high-dose methotrexate/ ifosfamide, etoposide and high dose cytarabin (CODOX$\mathrm{M} / \mathrm{IVAC}$ ) for human immunodeficiency virus-associated Burkitt lymphoma. Cancer 2003; 98: 1196-205.

33. Kaplan LD, Lee JY, Ambinder RF, Sparano JÁ, Cesarman E, Chadburn A, et al. Rituximab does not improve clinical outcome in a randomized phase 3 trial of CHOP with or without rituximab in patients with HIV.associated non-Hodgkin lymphoma: AIDS-malignancies Consortium Trial 010. Blood 2005; 106: 1538-43.

34. Oriol A, Ribera JM, Bergua J, Gimenez Mesa E, Grande C, Esteve J, et al. High dose chemotherapy and immunotherapy in adult Burkitt lymphoma: comparison of results in human immunodeficiency virus- infected and noninfected patients. Cancer 2008; 113: 117-25.

35. Balsalobre P, Diez-Martin JL, Re A, Michieli M, Ribera JM, Canals C, et al. Autologous stem-cell transplantation in patients with HIV-related lymphoma. J Clin Oncol 2009; 27: 2192-8.

36. Hoffmann C, Wolf E, Factkenheuer G, Buhk T, Stoehr A, Plettenberg A, et al. Response to highly active antiretroviral therapy strongly predicts outcome in patients with AIDS-related lymphoma. AIDS 2003; 17: 1521-9. 